Retraction

\title{
Retracted: N-(1-Pyrenyl) Maleimide Induces Bak Oligomerization and Mitochondrial Dysfunction in Jurkat Cells
}

\author{
BioMed Research International \\ Received 2 September 2021; Accepted 2 September 2021; Published 28 October 2021 \\ Copyright (c) 2021 BioMed Research International. This is an open access article distributed under the Creative Commons \\ Attribution License, which permits unrestricted use, distribution, and reproduction in any medium, provided the original work \\ is properly cited.
}

BioMed Research International has retracted the article titled "N-(1-Pyrenyl) Maleimide Induces Bak Oligomerization and Mitochondrial Dysfunction in Jurkat Cells" [1] due to concerns regarding the reliability of the results. The journal has been made aware [2] that the article includes nucleotide sequences which appear to be incorrectly targeted, as follows:

- CTTCGTACCATTTTTG targets ZHX3 mRNA, not Bak as described in the article

- TGTGTGAAAGTTTTTG targets YWHAEP7 noncoding RNA, not Bid as described in the article

- TTGGCAACCGCTTTTTG targets Luciferase 1 in P. pyralis, but only with $93 \%$ identity

The authors did not respond to our requests for clarification, and due to the above concerns, it is being retracted with the agreement of the journal and the editorial board.

\section{References}

[1] P.-R. Huang, S.-C. Hung, C.-C. Pao, and T.-C. V. Wang, "N(1-Pyrenyl) Maleimide Induces Bak Oligomerization and Mitochondrial Dysfunction in Jurkat Cells," BioMed Research International, vol. 2015, Article ID 798489, 10 pages, 2015.

[2] C. Labbé, N. Grima, T. Gautier, B. Favier, and J. A. Byrne, "Semi-automated fact-checking of nucleotide sequence reagents in biomedical research publications: the Seek \& Blastn tool," PLoS One, vol. 14, no. 3, 2019. 


\title{
N-(1-Pyrenyl) Maleimide Induces Bak Oligomerization and Mitochondrial Dysfunction in Jurkat Cells
}

\author{
Pei-Rong Huang, ${ }^{1}$ Shu-Chen Hung, ${ }^{2}$ Chia-Chu Pao, ${ }^{3}$ and Tzu-Chien V. Wang ${ }^{1,3}$ \\ ${ }^{1}$ Department of Molecular and Cellular Biology, College of Medicine, Chang Gung University, Kwei-San, Tao-Yuan 333, Taiwan \\ ${ }^{2}$ Health Sciences Research Institute and School of Natural Sciences, University of California, Merced, CA 95343, USA \\ ${ }^{3}$ Institute of Biomedical Sciences, College of Medicine, Chang Gung University, Kwei-San, Tao-Yuan 333, Taiwan
}

Correspondence should be addressed to Tzu-Chien V. Wang; tcvwg@mail.cgu.edu.tw

Received 10 November 2014; Revised 20 December 2014; Accepted 20 December 2014

Academic Editor: Sergio Lavandero

Copyright (C) 2015 Pei-Rong Huang et al. This is an open access article distributed under the Creative Commons Attribution License, which permits unrestricted use, distribution, and reproduction in any medium, provided the original work is properly cited.

$\mathrm{N}$-(1-pyrenyl) maleimide (NPM) is a fluorescent reagent that is frequently used as a derivatization agent for the detection of thiocontaining compounds. NPM has been shown to display a great differential cytotoxicity against hematopoietic cancer cells. In this study, the molecular mechanism by which NPM induces apoptosis was examined. Here, we show that treatment of Jurkat cells with NPM leads to Bak oligomerization, loss of mitochondrial membrane potential $(\Delta \psi m)$, and release of cytochrome C from mitochondria to cytosol. Induction of Bak oligomerization appears to play a critical role in NPM-induced apoptosis, as downregulation of Bak by shRNA significantly prevented NPM-induced apoptosis. Inhibition of caspase 8 by Z-IETD-FMK and/or depletion of Bid did not affect NPM-induced oligomerization of Bak. Taken together, these results suggest that NPM-induced apoptosis is mediated through a pathway that is independent of caspase- 8 activation.

\section{Introduction}

Apoptosis, the programmed cell death type I, plays an important role in developmental processes, maintenance of homeostasis, and elimination of damaged cells. One pathway of apoptosis is known to be triggered by the release of cytochrome $\mathrm{C}$ from mitochondria to cytosol, which activates the caspase cascade through the formation of a caspaseactivating protein complex called the apoptosome $[1,2]$. The release of cytochrome $\mathrm{C}$ is regulated by anti- and proapoptotic $\mathrm{Bcl}-2$ family of proteins, which reside in the outer membrane of mitochondria [3]. The ratio of proapoptotic to antiapoptotic BCL-2 family proteins is a critical determinant of cytochrome $\mathrm{C}$ release. When the $\mathrm{BH} 3$-only proteins $\mathrm{tBid}$ and Bim are able to engage the effectors Bak and Bax, Bax and/or Bak undergoes a conformational change, leading to its oligomerization $[4,5]$. The pore-forming capability of the oligomerized Bax and/or Bak results in destabilization of the mitochondrial outer membrane and subsequent release of cytochrome $\mathrm{C}$ from the mitochondria $[6,7]$.
Among the biological capabilities that are acquired during the development of human tumors, enhanced resistance to apoptosis is common in most cancers [8]. Tumor cells have evolved a variety of strategies to limit or circumvent apoptosis, including activation or enhanced expression of antiapoptotic factors (such as BCL-2, BCL-XL, and IAPs), inactivation of the master proapoptotic p53 pathway thus preventing upregulation of proapoptotic BCL-2 family proteins upon genotoxic stress $[9,10]$. The induction of apoptotic cell death represents the mechanism of action for many anticancer drugs. In fact, most cytotoxic agents currently used in cancer therapy appear to exert their cytotoxic action through apoptosis induction (reviewed in [8]).

During a search for anticancer drugs by screening for inhibitors of telomerase, we have identified several small molecule inhibitors, including helenalin [11] and maleimide-derived compound U-73122 [12] and N-(1pyrenyl) maleimide (NPM) [13], that selectively inhibit telomerase in a cell-free system. One common feature of these compounds is that they are all thio-directed 
covalent modification agents. Although the mechanism for the selective inhibition of telomerase by these agents is presently not known, the compound NPM has been shown to display the greatest differential cytotoxicity against hematopoietic cancer cells. Surprisingly, however, through inhibition of telomerase and potentially modulation of uncharacterized targets, the primary cytotoxic effect of NPM against hematopoietic cancer cells manifests by induction of apoptosis [13]. In this study, the molecular mechanism by which NPM induces apoptosis was examined in order to advance our basic understanding for the potential application of this drug in the treatment of leukemia.

\section{Materials and Methods}

2.1. Chemicals, Enzymes, Antibodies, and Oligonucleotides. Fetal bovine serum (FBS), Superscript III reverse transcriptase, TRIzol reagent, and antibiotics were from Gibco-BRL. Taq DNA polymerase was from ABgene, Surrey, UK. Antibodies against caspases 3 (9662), 7 (9492), 8 (9746), PARP (9542), Bim (2933), Bid (2002), and Bak (6947) were purchased from Cell Signaling Technology. Antibodies against Bad (610391) and Bcl-2 (610538) were purchased from BD Biosciences. Antibody against $\mathrm{Bcl}-\mathrm{X}_{\mathrm{L}}$ (633901) was purchased from BioLegend. Anti-actin (MAB1501) antibody was purchased from Millipore. Broad-spectrum caspase inhibitor zVAD-fmk was purchased from Promega. Caspase- 8 inhibitor Z-IETD-FMK was purchased from BD Pharmingen. Gel electrophoresis reagents were from Bio-Rad. N-(1-pyrenyl) maleimide and other chemicals were from Sigma Chemical Co.

2.2. Cell Culture. Acute T cell leukemia-derived Jurkat (clone E6.1) cells were cultured in RPMI 1640 medium containing 10\% FBS, 2 mM L-glutamine, $4.5 \mathrm{~g} / \mathrm{L}$ glucose, $10 \mathrm{mM}$ HEPES, $1.0 \mathrm{mM}$ sodium pyruvate, and antibiotics $(100 \mathrm{U} / \mathrm{mL}$ penicillin, $100 \mathrm{U} / \mathrm{mL}$ streptomycin, and $0.25 \mu \mathrm{g} / \mathrm{mL}$ amphotericin B). Human promyelocytic leukemia HL60 cells were cultured in Iscove's modified Dulbecco's medium containing 20\% FBS, $4 \mathrm{mM}$ L-glutamine, and antibiotics. Human embryo kidney cells HEK293T were cultured in DMEM supplemented with $10 \%$ fetal bovine serum, $2 \mathrm{mM}$ L-glutamine, and antibiotics. All cells were grown at $37^{\circ} \mathrm{C}$ in a humidified incubator containing $5 \% \mathrm{CO}_{2}$.

2.3. Measurement of Mitochondrial Membrane Potential $(\Delta \psi m)$ Loss by Flow Cytometry. Membrane-permeable lipophilic cationic fluorochrome JC-1 $\left(5,5^{\prime}, 6,6^{\prime}\right.$-tetrachloro$1,1^{\prime}, 3,3^{\prime}$-tetraethylbenzimidazolcarbocyanine iodide), which exhibits potential-dependent accumulation in the mitochondria, was used to evaluate the status of $\Delta \psi m$ according to the instruction in Flow Cytometry Mitochondrial Membrane Potential Detection Kit (BD Biosciences). In brief, $10^{6}$ cells were washed and resuspended in $500 \mu \mathrm{L}$ of JC-1 working solution. After incubating at $37^{\circ} \mathrm{C}$ for $15 \mathrm{~min}$, cells were harvested and washed with Assay Buffer twice. The change in $\Delta \psi m$ was analyzed by flow cytometry (BD FACSCalibur TM system, Becton Dickinson) using $488 \mathrm{~nm}$ Argon ion laser for excitation. The emitted green and red fluorescence light of
JC-1 were detected by using filters 530/30 nm and 585/42 nm, respectively. Data analysis was performed with BD Cell Quest pro software (Becton Dickinson).

2.4. Detection of Cytochrome C Release. The release of cytochrome $\mathrm{C}$ from the mitochondria was assayed by FlowCellect Cytochrome C kit (Millipore). In brief, $2 \times 10^{5}$ cells were washed and resuspended in $200 \mu \mathrm{L}$ of permeabilization buffer. After incubating on ice for $10 \mathrm{~min}, 200 \mu \mathrm{L}$ of the fixation buffer was added to each sample and incubated at room temperature for $20 \mathrm{~min}$. The permeabilized cells were collected by centrifugation and then resuspended in $200 \mu \mathrm{L}$ of blocking buffer. After incubating at $25^{\circ} \mathrm{C}$ for $30 \mathrm{~min}, 20 \mu \mathrm{L}$ of anti-cytochrome C-FITC antibody was added to each sample and incubated at $25^{\circ} \mathrm{C}$ for $30 \mathrm{~min}$ in the dark. The cell mixture was washed with blocking buffer and a total of 10,000 cells were analyzed by flow cytometry (BD FACSCaliburTM system, Becton Dickinson) using $488 \mathrm{~nm}$ Argon ion laser for excitation. The emitted green (FITC) light was detected by using filters $530 / 30 \mathrm{~nm}$. Data analysis was performed with BD Cell Quest pro software (Becton Dickinson).

2.5. Determination of Bak and Bax Oligomerization. In vitro crosslinking for the detection of Bak and Bax oligomerization was performed according to the methods of Shawgo et al. [14]. In brief, $10^{6}$ cells were incubated in $80 \mu \mathrm{L}$ of PBS containing $100 \mathrm{mM}$ EDTA and $1 \mathrm{mM}$ cross-linking agent bismaleimidohexane $(\mathrm{BMH})$ at $22^{\circ} \mathrm{C}$ for $30 \mathrm{~min}$. Following incubation, cells were quenched with $100 \mathrm{mM}$ dithiothreitol for $15 \mathrm{~min}$ and pelleted by centrifugation. The pelleted cells were suspended in RIPA lysis buffer $(25 \mathrm{mM}$ Tris. $\mathrm{HCl}, \mathrm{pH}$ 7.6, $150 \mathrm{mM} \mathrm{NaCl}, 1 \% \mathrm{NP}-40,1 \%$ sodium deoxycholate, $0.1 \%$ SDS), and the detection of Bak and Bax oligomers were assayed by Western blot.

2.6. Plasmids. The plasmids pMD.G, pCMVdelR8.91, and pLKO.1 were purchased from National RNAi Core Facility at Academia Sinica of Taiwan. The plasmids pMD.G and pCMVdelR8.91 were used for the packaging of lentiviral particles. The plasmids that express shRNA to knock down Bak and Bid were constructed by inserting the following sequences into the lentiviral vector pLKO.1. The selected shRNA sequences for Bak and Bid were $5^{\prime}$-CTTCGTACCATTTTTG- $3^{\prime}$ and $5^{\prime}$-TGTGTGAAAGTTTTTG$3^{\prime}$, respectively. The shRNA sequence $5^{\prime}$-TTGGCAACCGCTTTTTG-3', which targets firefly luciferase mRNA, served as the control.

2.7. Lentivirus Production, Infection, and Selection of shRNAExpressing Cells. To generate lentiviral particles, HEK293T cells were cotransfected with lentiviral plasmid and packaging plasmids (pMD.G and pCMVdelR8.91) by Arrest-In (Open Biosystems) according to manufacturer's instruction. After $16 \mathrm{~h}$ of transfection, media containing the transfection reagents were removed and replenished with fresh media. After incubating for another $48 \mathrm{~h}$, the media containing viral particles were centrifuged at $1250 \mathrm{rpm}$ for $5 \mathrm{~min}$, and the supernatants were collected for the infection studies. Infection of cells with lentiviral particles was carried out at 


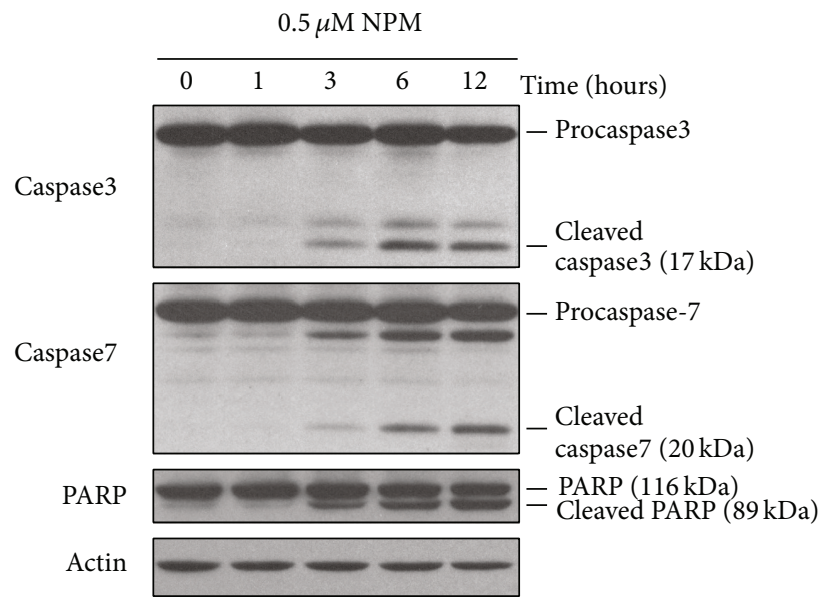

(a)

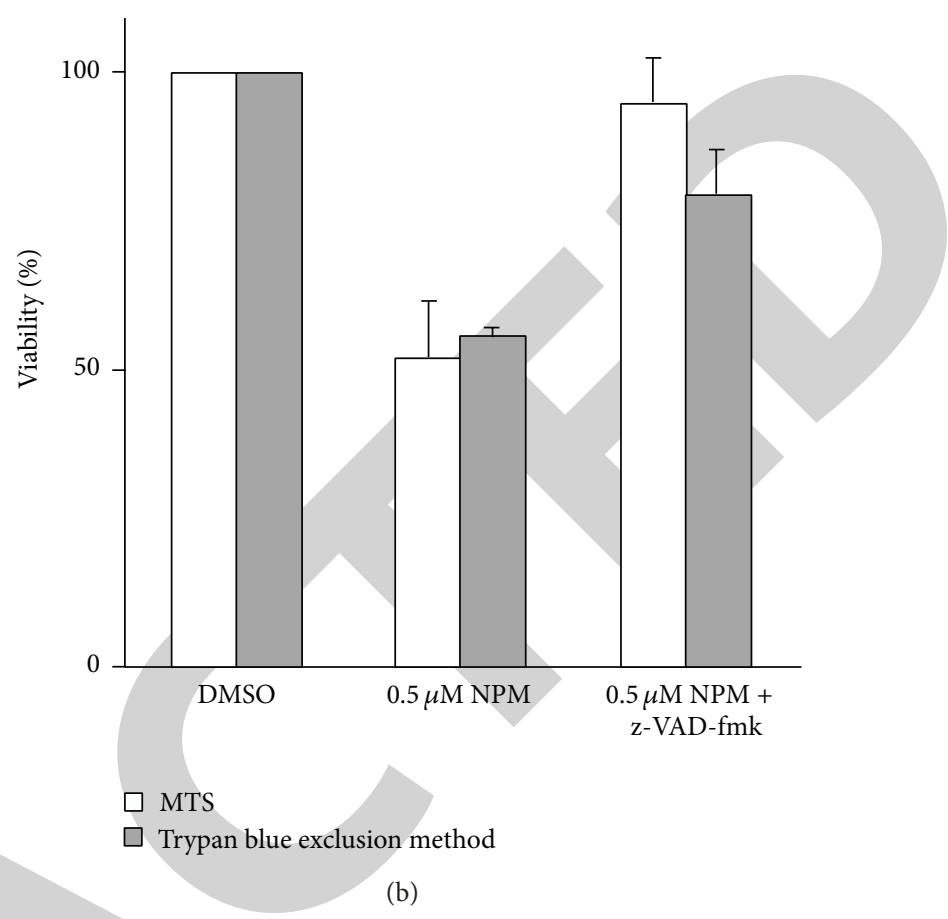

FIGURE 1: NPM-induced apoptosis in Jurkat cells is dependent on caspase activation. (a) To determine the onset of caspase activation, Jurkat cells were treated with $0.5 \mu \mathrm{M}$ NPM for the indicated time. The cell lysates were analyzed for the cleavage of caspases 3,7 , and PARP by Western blot. Actin served as a loading control. Data shown were from a representative of three independent experiments that gave similar results. (b) Effect of caspase inhibition on the viability of NPM-treated cells. Jurkat cells were pretreated with the broad-spectrum caspase inhibitor z-VAD-fmk $(50 \mu \mathrm{M})$ for $1 \mathrm{~h}$ before exposing to $0.5 \mu \mathrm{M}$ of NPM for $24 \mathrm{~h}$. Cell viability was then determined by MTS assay and trypan blue exclusion method as described in Section 2. Data shown for MTS assay are the means \pm SD from four independent experiments. Data shown for trypan blue exclusion method are the means \pm SD from three independent experiments.

$37^{\circ} \mathrm{C}$ in the presence of polybrene at $8 \mu \mathrm{g} / \mathrm{mL}$. Cells that stably express shRNA were obtained by culturing the infected cells in the presence of puromycin at $1 \mu \mathrm{g} / \mathrm{mL}$ for 1 week.

2.8. Assay for Viability. Viability of NPM-treated cells was determined by using trypan blue exclusion method and MTS assay. In brief, $10 \mu \mathrm{L}$ of cellular suspension was mixed with $10 \mu \mathrm{L}$ Trypan blue $(0.05 \%)$, and the percentage of nonviable cells (stained blue) was determined by microscopic examination in a hemocytometer. MTS assay was performed according to the manufacturer's suggestions (CellTiter $96^{\mathrm{R}}$ $\mathrm{AQ}_{\text {ueous }}$ One Solution Cell Proliferation Assay kit, Promega).

2.9. Western Blot. Western blot analysis was performed as previously described [15].

\section{Results}

3.1. NPM Induces Caspase Activation to Trigger Apoptosis. To confirm that NPM-induced cell death is associated with the activation of caspases, the kinetics of caspase activation as well as the effect of caspase inhibition on viability were analyzed in Jurkat cells. As shown in Figure 1(a), the cleavage of caspase-3, caspase-7, and PARP was readily detected as early as $3 \mathrm{~h}$ after exposure to $0.5 \mu \mathrm{M}$ of NPM. Pretreatment of the Jurkat cells with a broad-spectrum caspase inhibitor, zVAD-fmk, completely inhibited the cytotoxic effect of NPM
(Figure 1(b)), indicating that caspase activation plays a critical role in NPM-induced cell death.

3.2. NPM Induces Mitochondrial Dysfunction. To determine if the apoptosis induced by NPM is mediated by activation of the mitochondrial pathway, the effects of NPM treatment on mitochondrial membrane potential $(\Delta \psi m)$ and cytochrome $\mathrm{C}$ released from mitochondria were examined. For evaluation of changes in mitochondrial membrane potential $(\Delta \psi m)$, we used a membrane permeable dye, JC-1 (5,5' ,6, $6^{\prime}$-tetrachloro-1, $1^{\prime}, 3,3^{\prime}$ tetraethylbenzimidazolylcarbocyanine iodide), which exhibits potential-dependent accumulation in the mitochondria. In nonapoptotic cells with intact mitochondria, most of JC-1 exist as aggregates in the mitochondria and are detected as red fluorescence light ( $\mathrm{R} 1$ as shown in Figure 2(a)). When the mitochondrial membrane potential collapses, the JC-1 cannot accumulate within the mitochondria and exists as monomers in the cytosol, which are detected as green fluorescence light ( $\mathrm{R} 2$ as shown in Figure 2(a)). Therefore, loss of $\Delta \psi m$ can be determined by the ratios of JC-1 as aggregates (R1) or as monomers (R2). As shown in Figure 2(a), when the Jurkat cells were treated with $50 \mu \mathrm{M}$ CCCP (mitochondrial membrane potential disrupter), essentially all of JC-1 was detected as monomers (R2). Quantitative analysis of $\Delta \psi m$ as measured by the decrease of JC-1 aggregates (R1) indicated that loss of $\Delta \psi m$ was detectable 

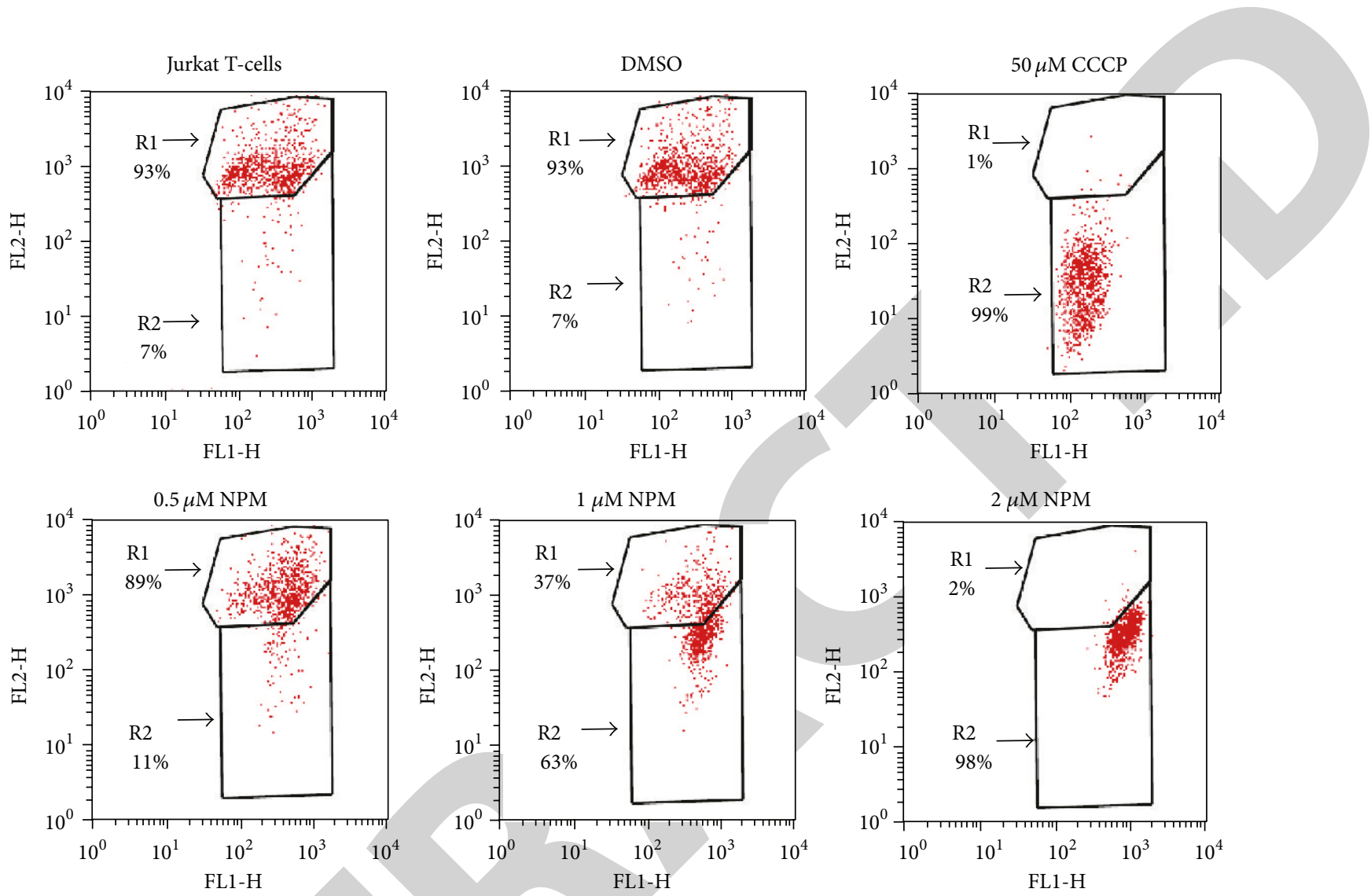

(a)
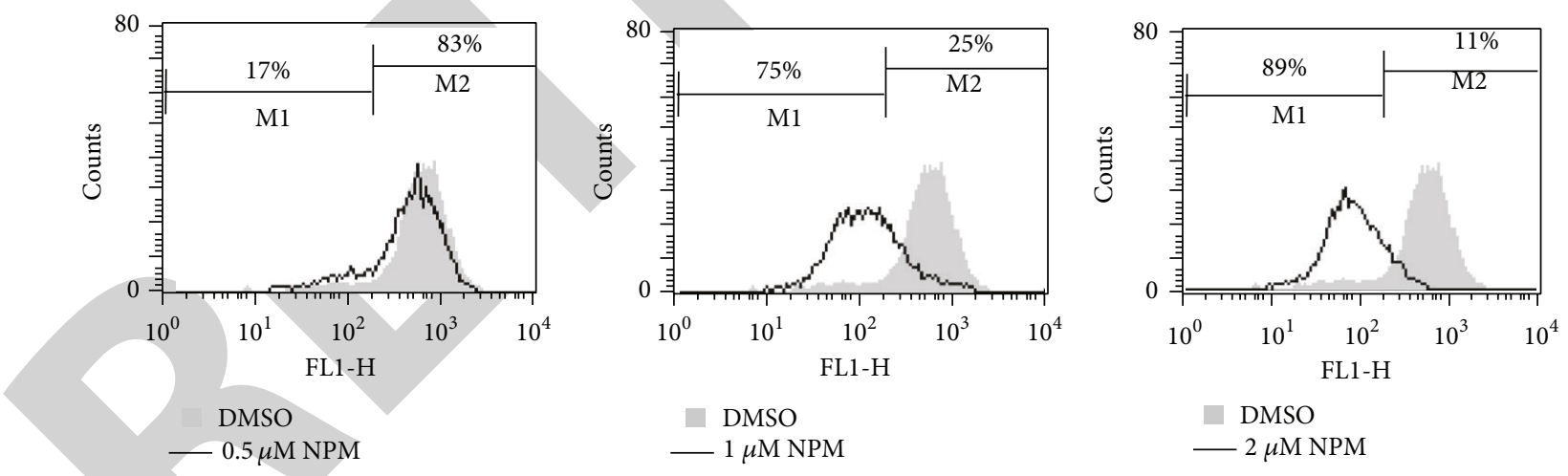

(b)

FIGURE 2: Effect of NPM on mitochondrial dysfunction in Jurkat cells. (a) Effect of NPM on the loss of mitochondrial membrane potential $(\Delta \psi m)$. Jurkat cells were treated with $50 \mu \mathrm{M}$ of CCCP (mitochondrial membrane potential disrupter) or $0.5-2 \mu \mathrm{M}$ of NPM for $3 \mathrm{~h}$. The treated cells were stained with JC-1 (5,5',6,6' -tetrachloro-1,1' $3,3^{\prime}$ tetraethylbenzimidazolylcarbocyanine iodide) before being analyzed for the loss of mitochondrial membrane potential $(\Delta \psi m)$ by flow cytometry as described in Section 2. FL1-H: JC-1 green fluorescence. FL2-H: JC-1 red fluorescence. R1 and R2 are the percentage of cells with polarized $\Delta \psi m$ and depolarized $\Delta \psi m$, respectively. (b) Effect of NPM on the release of cytochrome $\mathrm{C}$ from mitochondria to cytosol. Jurkat cells were treated with $0.5-2 \mu \mathrm{M}$ of NPM or DMSO as control for $3 \mathrm{~h}$. The treated cells were labeled with Anti-Cytochrome C-FITC antibody and analyzed by flow cytometry. FL1-H: cytochrome C-FITC. M1 and M2 are the percentage of cells with low (mitochondria that have released cytochrome C) and high (intact mitochondria) fluorescence, respectively. The M1 and M2 for the untreated cells (DMSO) are 6.6\% and 93.4\%, respectively. Data were from a representative of three independent experiments that gave similar results. 


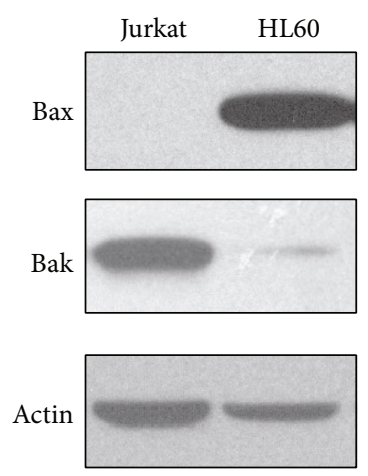

(a)

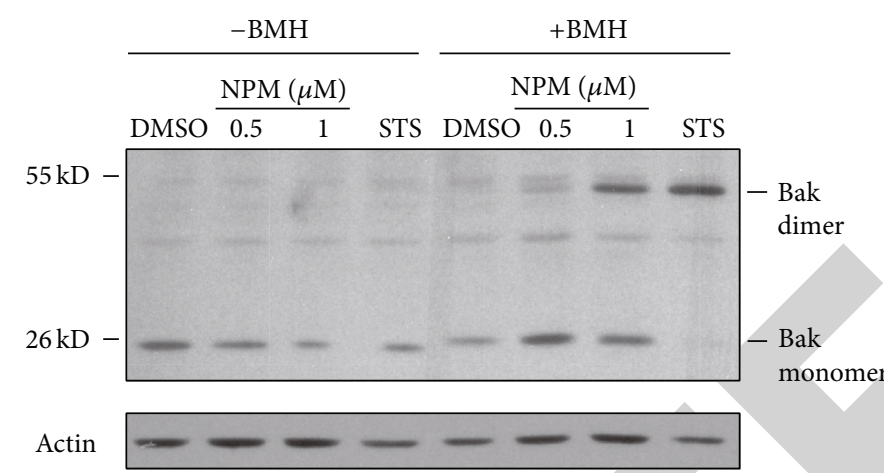

(b)

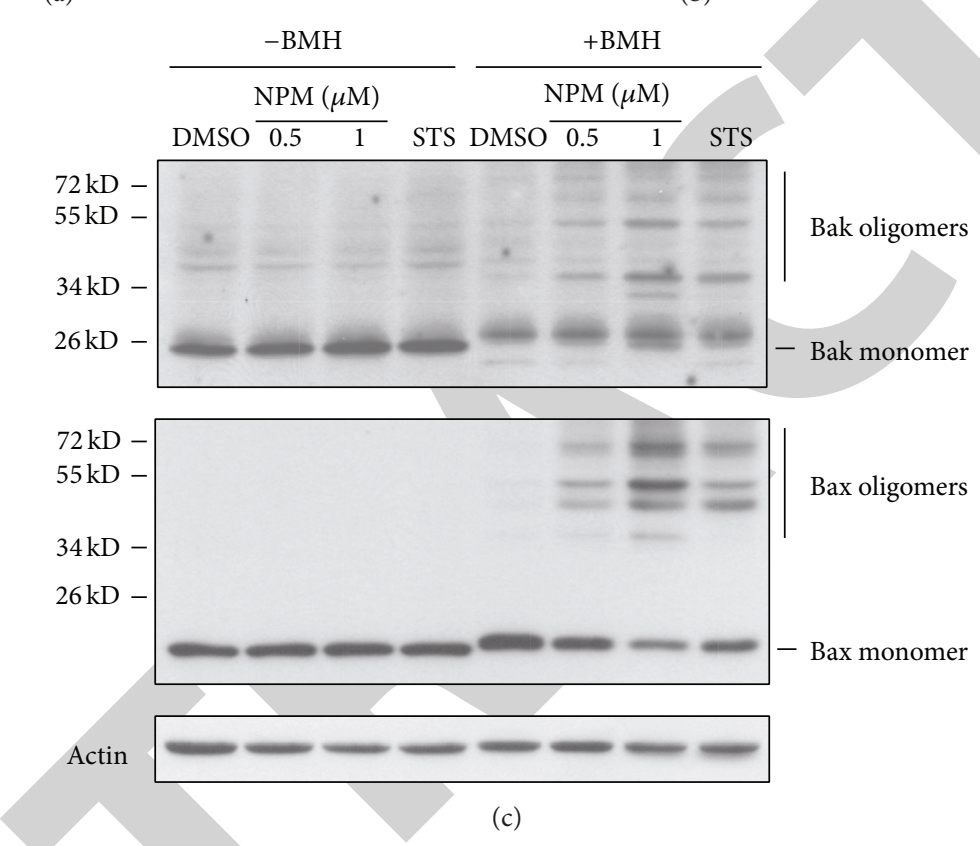

FIGURE 3: NPM induces oligomerization of Bak and Bax. (a) Expression of Bax and Bak in Jurkat and HL60 cells. The cell lysates of Jurkat and HL60 cells were analyzed for the expression of Bax and Bak by Western blot. The effect of NPM on the oligomerization of Bak and Bax in Jurkat (b) and HL60 (c) cells was analyzed by treating the cells with NPM at $0.5-1 \mu \mathrm{M}$ or with staurosporine (STS) at $500 \mathrm{nM}$ for $3 \mathrm{~h}$. The treated cells were assayed for the presence of Bax and Bak oligomers as described in Section 2. Data shown were from a representative of two independent experiments that gave similar results. Actin served as a loading control. BMH, bismaleimidohexane.

in the Jurkat cells treated with $0.5 \mu \mathrm{M}$ of NPM and was greatly increased in the cells treated with 1-2 $\mu \mathrm{M}$ of NPM.

Because loss of $\Delta \psi m$ is often associated with the release of mitochondrial intermembrane space proteins into the cytosol, we next investigated whether cytochrome $\mathrm{C}$ is released from the NPM-treated cells. As shown in Figure 2(b), the percentage of cells with low fluorescence (M1) increased from $6.6 \%$ in the untreated control to $17 \%, 75 \%$, and $89 \%$ in the cells treated with $0.5,1$, and $2 \mu \mathrm{M}$ of NPM, indicating that cytochrome $\mathrm{C}$ is released from mitochondria in the cells treated with NPM.

3.3. NPM Induces Bak and Bax Oligomerization. Bax and Bak play a critical role in triggering mitochondria-mediated apoptosis $[16,17]$. Since the Jurkat (clone E6.1) cells used in this study do not express Bax ([14] and Figure 3(a)) due to frameshift mutation resulting in premature termination of translation [18], we examined whether or not oligomerization of Bak is involved in the NPM-induced apoptosis. To address this issue, Jurkat cells were exposed to varying concentrations of NPM or $500 \mathrm{nM}$ of staurosporine (as a positive control for inducing Bak oligomerization) for $3 \mathrm{~h}$ and then treated with membrane-permeable protein cross-linker BMH. As shown in Figure 3(b), NPM induced the formation of Bak homodimer $(52 \mathrm{kDa})$, but not higher order Bak oligomers, in a concentration-dependent manner in Jurkat cells. To address if the lack of higher Bak oligomerization was unique to the Jurkat cells used in this study, we examined the induction of Bax and Bak oligomerization in HL60 cells. As shown in Figure 3(c), NPM and staurosporine induced the formation of higher oligomers of Bak and Bax in HL60 cells.

3.4. Suppression of NPM-Induced Apoptosis by Downregulation of Bak. To clarify the role of Bak in NPM-induced apoptosis, we examined the effects of Bak depletion on NPMinduced mitochondrial dysfunction and caspase activation. 


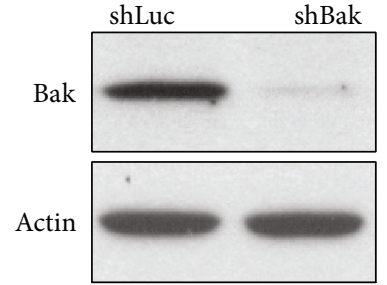

(a)

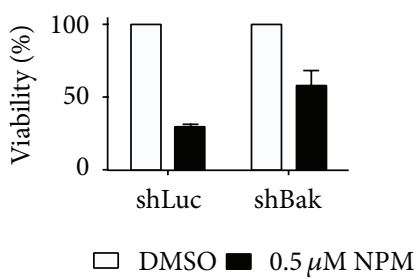

(b)
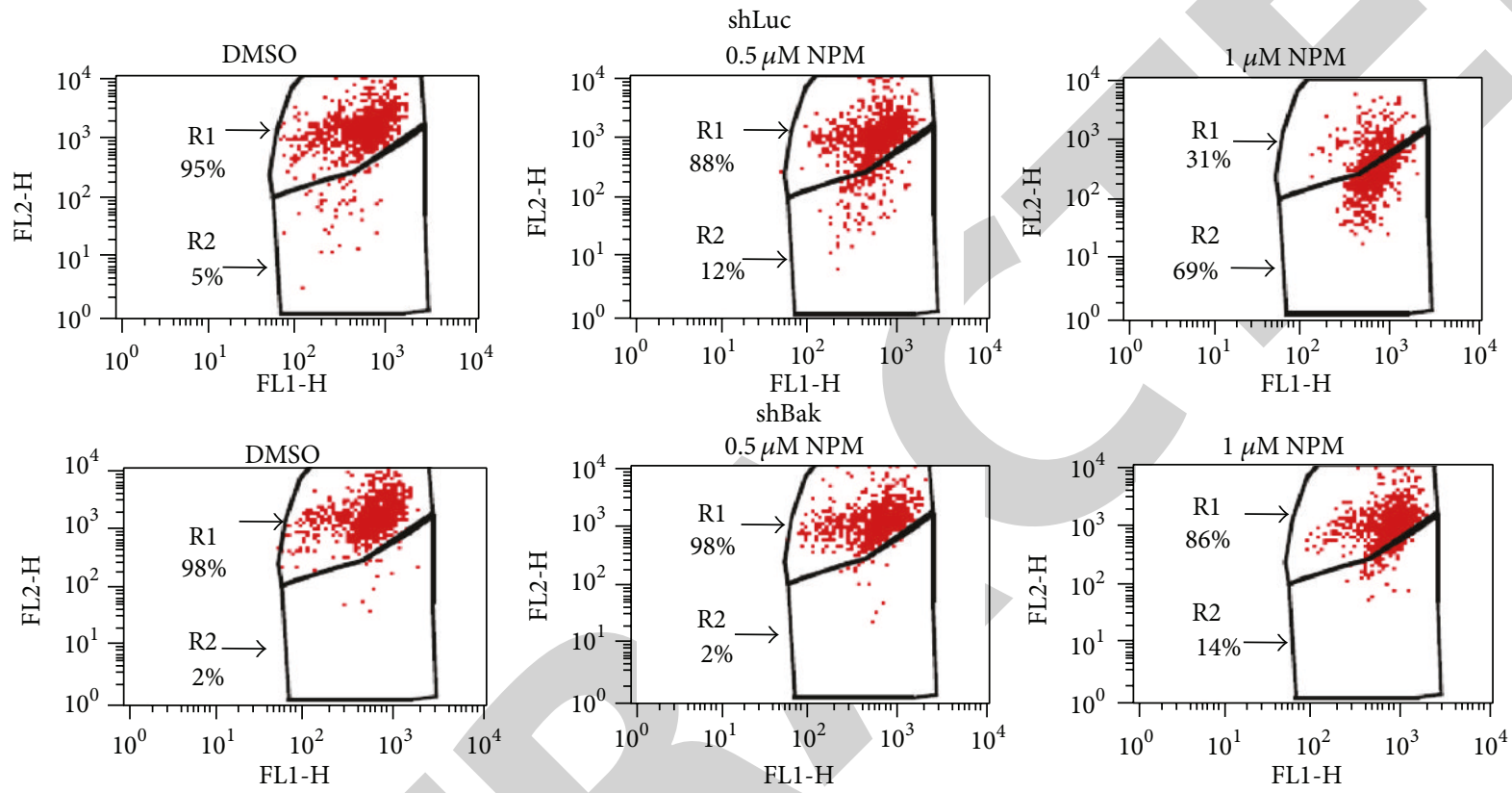

(c)

shLuc
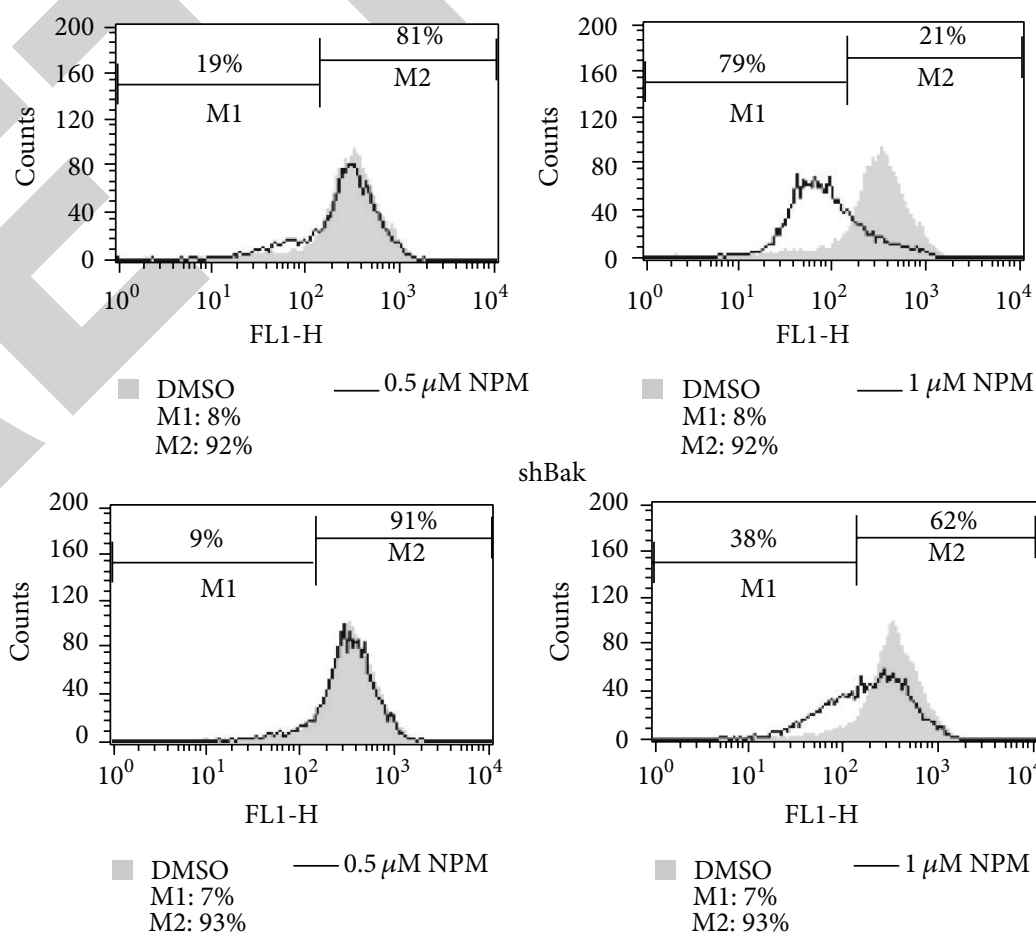

shBak

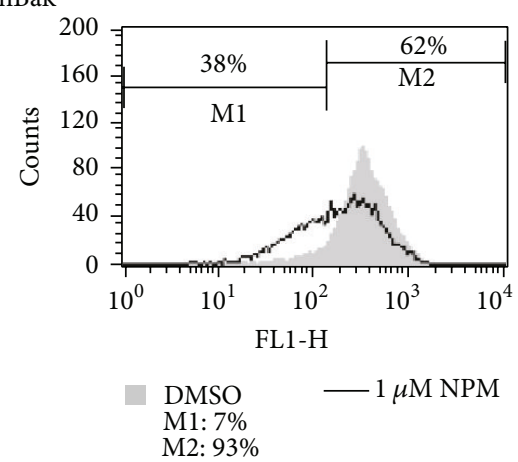

(d)

FIgUre 4: Continued. 


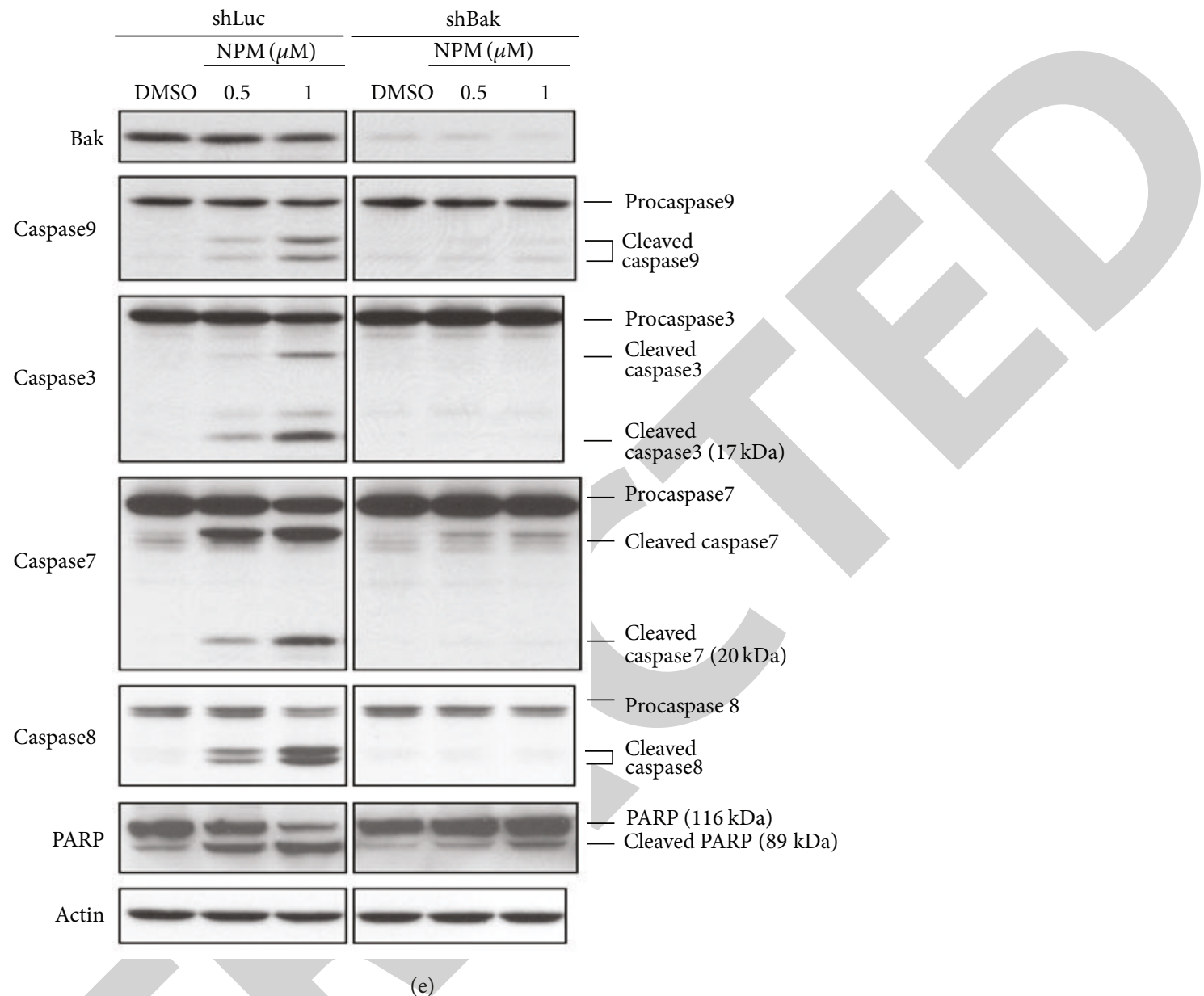

(e)

Figure 4: Effect of Bak knockdown on NPM-induced apoptosis in Jurkat cells. (a) Expression of Bak in the Jurkat cells that stably express shRNA against Bak (shBak) or against luciferase (shLuc). Expression of Bak in the cell lysates was assayed by Western blot. The effect of Bak knockdown on NPM-induced cytotoxicity (b), loss of mitochondrial membrane potential $(\Delta \psi m)(\mathrm{c})$, the release of cytochrome C from mitochondria to cytosol (d), and caspase activation (e) were analyzed in the Jurkat cells that stably express shRNA against Bak (shBak) or against luciferase (shLuc), as described in the legends to Figures 1 and 2. Data were from a representative of three independent experiments that gave similar results.

Jurkat cells were infected with shRNA-expressing lentiviral particles and the cells that stably express shRNA to knock down Bak were examined for NPM-induced loss of mitochondrial membrane potential $(\Delta \psi m)$, cytochrome C release, and caspase activation. As shown in Figure 4(a), the expression level of Bak was greatly reduced in the cells that stably express shRNA against Bak (shBak) as compared to cells that express shRNA against luciferase (shLuc). Depletion of Bak resulted in an increased resistance to NPM treatment (Figure 4(b)) and significantly suppressed the NPMinduced loss of $\Delta \psi m$ (Figure $4(\mathrm{c})$ ), cytochrome $\mathrm{C}$ release (Figure 4(d)), and caspase activation (Figure 4(e)), indicating that Bak plays an important role in NPM-induced apoptosis in Jurkat cells.

3.5. Effect of NPM on the Levels of Bcl-2 Family Members. To evaluate the role of $\mathrm{Bcl}-2$ family members in governing NPM-induced release of cytochrome $\mathrm{C}$ from mitochondria, the expression levels of proapoptotic (Bad, Bim, Bid, and Bak) and antiapoptotic (Bcl-2, Bcl-xL and $\mathrm{Mcl}-1) \mathrm{Bcl}-2$ proteins were examined in detail. Although the level of $\mathrm{Bcl}-2$ has been reported to be slightly reduced in the NPM-treated cells [13], more detailed analyses, however, have indicated that there is no significant reduction of $\mathrm{Bcl}-2$ in the NPM-treated cells. As shown in Figure 5(a), the levels of Bcl-2 and two other antiapoptotic proteins, $\mathrm{Bcl}-\mathrm{xL}$ and $\mathrm{Mcl}-1$, were not noticeably changed in the NPM-treated cells. While the proapoptotic proteins Bad and Bak were not noticeably changed, the levels of $\mathrm{tBid}$ and $\mathrm{tBim}_{\mathrm{EL}}$ were increased (Figure 5(b)). Kinetic analysis for the increased detection of tBid revealed that the increased tBid was readily detected in the cells treated with $0.5 \mu \mathrm{M}$ of NPM for $3 \mathrm{~h}$ and was accompanied with the activation of caspase 8 (Figure 5(c)). In contrast, the level of $\mathrm{tBim}_{\mathrm{EL}}$ appeared to increase only at a later time (Figure $5(\mathrm{~d})$ ).

3.6. Caspase-8 Activation Is Not Involved in NPM-Induced Bak Oligomerization. Since it is known that the ligation of death receptors in the extrinsic pathway can cause the activation of caspase 8 and cleavage of Bid, leading to the activation of 


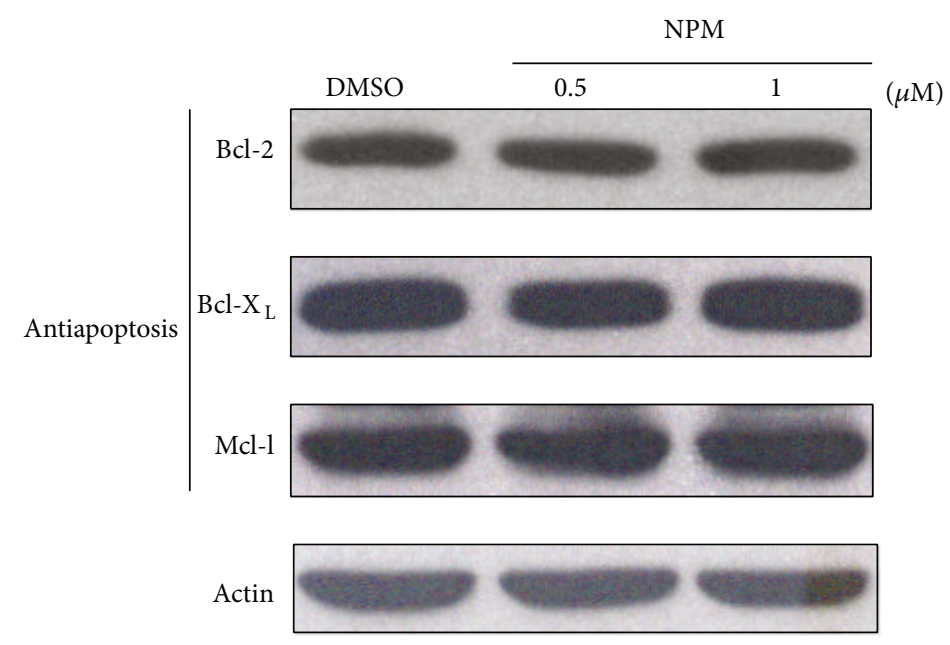

(a)

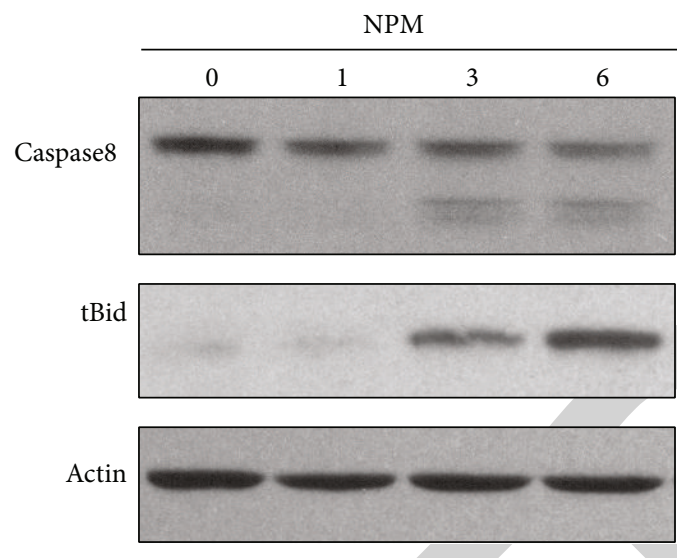

(c)

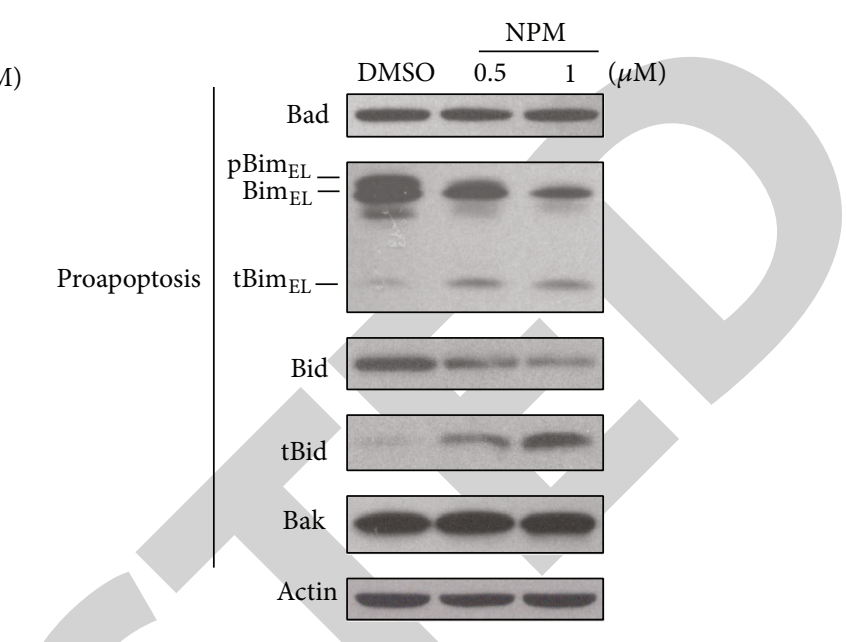

(b)

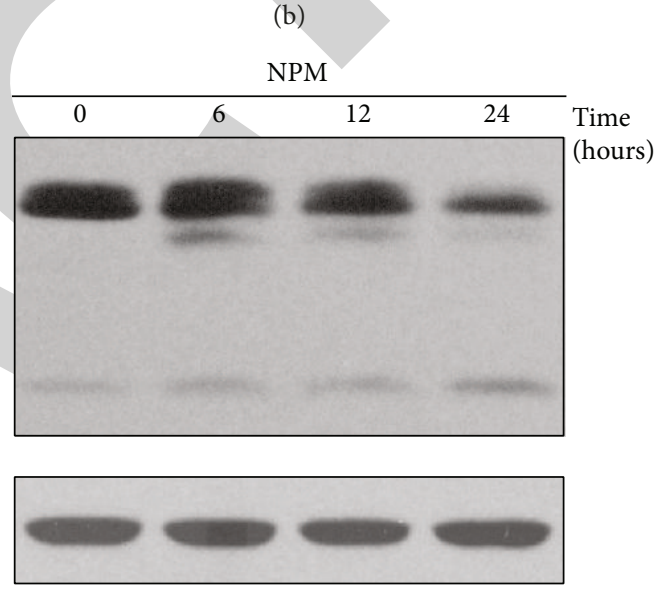

(d)

FIGURE 5: Effect of NPM on the levels of Bcl-2 family members and caspase 8 activation in Jurkat cells. Jurkat cells were treated with $0.5-1 \mu \mathrm{M}$ of NPM for $24 \mathrm{~h}$ and the cell lysates were analyzed for the levels of antiapoptotic Bcl-2 family proteins (Bcl-2, Bcl-XL, and Mcl-1) (a) and proapoptotic Bcl-2 family proteins (Bad, Bim, Bid, and Bak) (b) by Western blot. To monitor the levels of active cleaved caspase 8 , tBID, and BIM over time, Jurkat cells were analyzed by Western blot $((\mathrm{c})$ and $(\mathrm{d}))$. $\mathrm{tBid}$, truncated Bid; $\mathrm{Bim}_{\mathrm{EL}}$, Bim extra long isoform; $\mathrm{pBim}$ phosphorylated $\mathrm{Bim}_{\mathrm{EL}} ; \mathrm{tBim}_{\mathrm{EL}}$, truncated $\mathrm{Bim}_{\mathrm{EL}}$. Bim $\mathrm{BL}_{\mathrm{EL}}$ is one of the major isoforms produced by alternative splicing. Bim $\mathrm{EL}$ differs from $\mathrm{Bim}_{\mathrm{L}}$ by the inclusion of exon 3. Actin served as a loading control. Data were from a representative of three independent experiments that gave similar results.

Bak or Bax [1], we investigated whether or not activation of caspase 8 and cleavage of Bid is involved in NPM-induced oligomerization of Bak. Inhibition of caspase 8 with Z-IETDFMK abolished the cleavage of tBid but did not affect NPMinduced oligomerization of Bak (Figure 6(a)). Depletion of Bid by shRNA greatly reduced the level of Bid, but NPMinduced Bak oligomerization was not affected (Figure 6(b)). These results suggest that activation of caspase 8 and cleavage of Bid are not involved in NPM-induced oligomerization of Bak.

\section{Discussion}

NPM is a fluorescent reagent that is frequently used as a derivatization agent for the detection of thio-containing compounds $[19,20]$. While little is known about the biological effect of NPM, we have recently shown that NPM induces apoptosis and displays a great differential cytotoxicity against hematopoietic cancer cells [13], making this compound an attractive candidate to be tested for its anticancer activity in vivo. In this work, we demonstrated that the apoptosis induced by NPM is mediated by the activation of executioner caspases and can be fully prevented by the caspase inhibitor, z-VAD-fmk (Figure 1). NPM appears to induce apoptosis by promoting mitochondrial dysfunction in Jurkat cells, as evidenced by the loss of $\Delta \psi m$ and the release of cytochrome $\mathrm{C}$ from mitochondria (Figure 2).

Activation of Bak by a conformational change has been reported in the apoptosis induced by many agents [21]. A model for Bak oligomerization has been proposed [22]. In this model, activation of Bak in the mitochondria is postulated to lead to the exposure of its $\mathrm{BH} 3$ domain which inserts into the hydrophobic groove of an adjacent activated Bak molecule, resulting in the formation of a symmetrical 


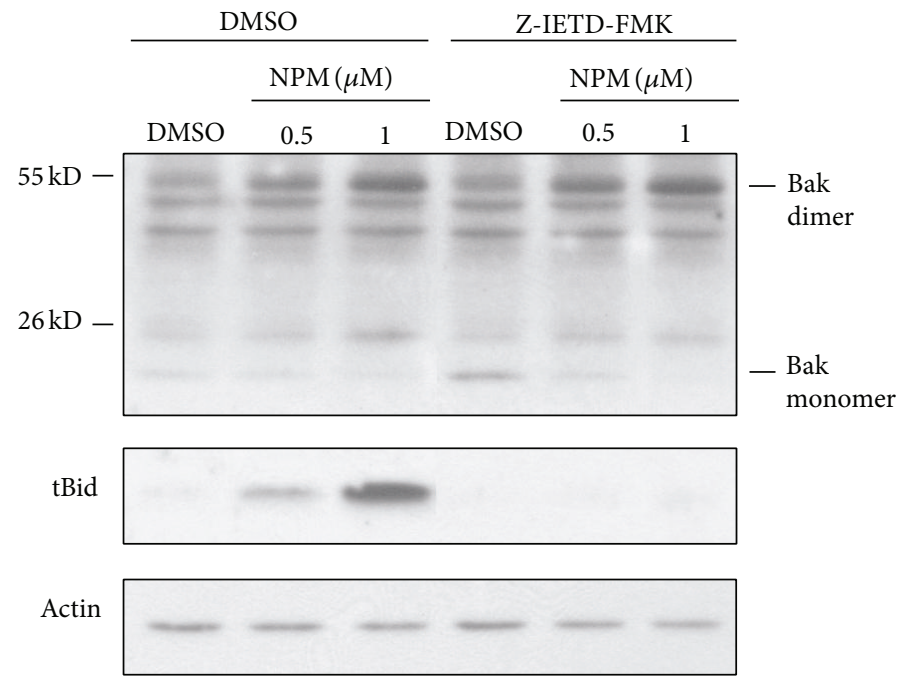

(a)

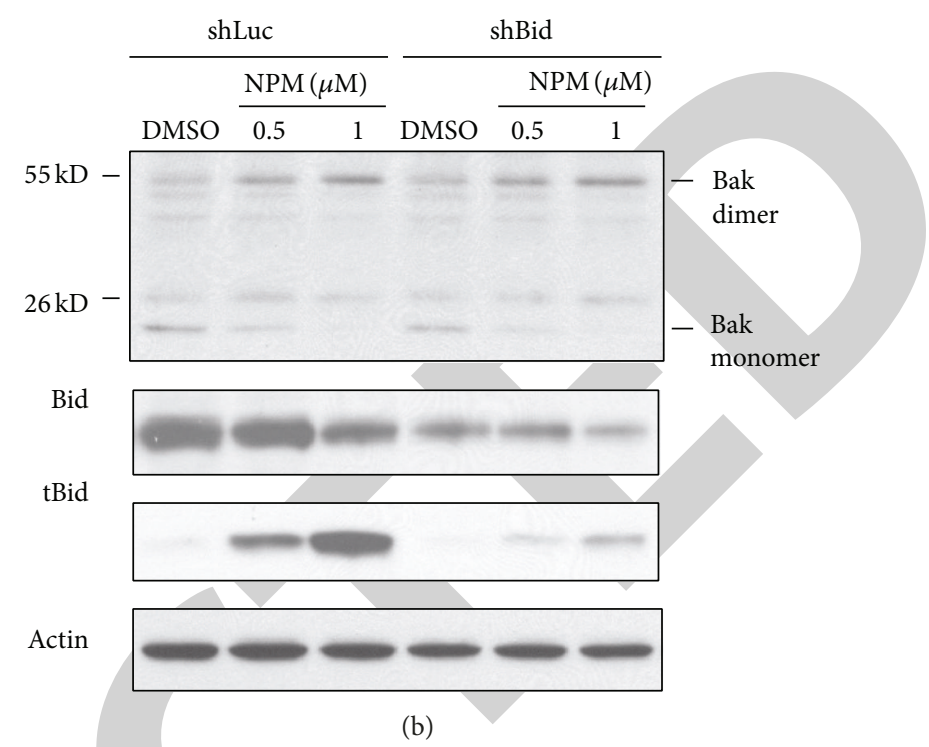

(b)

FIGURE 6: Effect of caspase-8 inhibition and Bid downregulation on NPM-induced Bak oligomerization. (a) Effect of caspase-8 inhibition on NPM-induced oligomerization of Bak and tBid formation. Jurkat cells were pretreated with $50 \mu \mathrm{M}$ of caspase-8 inhibitor Z-IETD-FMK for $1 \mathrm{~h}$ before being exposed to 0.5 or $1 \mu \mathrm{M}$ of NPM for $3 \mathrm{~h}$. The cell lysates were then assayed for the Bak oligomers and tBid as described in Section 2. (b) Effect of Bid downregulation on NPM-induced oligomerization of Bak and tBid formation. The Jurkat cells that stably express shRNA against Bid (shBid) or against luciferase (shLuc) were treated with NPM at $0.5-1 \mu \mathrm{M}$ for $3 \mathrm{~h}$. The cell lysates of the treated cells were assayed for the Bak oligomers and tBid as described in Section 2. Data shown were from a representative of three independent experiments that gave similar results. Actin served as a loading control.

Bak homodimer. Higher oligomers of Bak may be formed by dimer-dimer interactions mediated through a cryptic interface that is exposed following Bak activation [23]. Here, we demonstrated that NPM induces the oligomerization of Bak in both Jurkat and HL-60 cells (Figure 3). Activation of Bak appears to play an important role in NPM-induced apoptosis in Jurkat cells, as downregulation of Bak increases the viability of NPM-treated cells (Figure 4(b)) and suppresses the loss of $\Delta \psi m$ and the release of cytochrome $\mathrm{C}$ from mitochondria (Figures 4(c) and 4(d)).

At present, the mechanism leading to the activation of Bak by NPM remains unresolved. It is known that the ligation of death receptors in the extrinsic pathway can cause the activation of caspase 8 and cleavage of Bid to tBid. The cleaved tBid induces an allosteric activation and homooligomerization of Bak, which results in destabilization of the mitochondrial outer membrane and cytochrome $\mathrm{C}$ efflux [1]. Although the cleavage of Bid by caspase 8 has been shown to be an important mechanism leading to the activation of Bak or Bax in the extrinsic pathway of apoptosis [24], we observed that the depletion of Bid and/or inhibition of caspase- 8 by Z-IETD-FMK did not appear to affect the NPMinduced formation of Bak homodimer (Figure 6). These results suggest that NPM-induced apoptosis does not depend on the extrinsic pathway of caspase- 8 activation to cleave Bid for the activation of Bak. The observed cleavage of Bid to tBid in NPM-treated cells may occur as a consequence of NPM-induced Bak oligomerization and subsequent caspase activation. In support of such a postulate, depletion of Bak effectively abolished the activation of caspase- 8 by NPM
(Figure 4(e)). Taken together, our results suggest that NPMinduced apoptosis is mediated through induction of Bak oligomerization by a pathway that is independent of caspase8 activation.

\section{Conflict of Interests}

The authors declare that there is no conflict of interests regarding the publication of this paper.

\section{Acknowledgments}

This work was supported by Grants to Tzu-Chien V. Wang from the Chang Gung Memorial Hospital (CMRPD1A0421 and CMRPD3D0171) and the National Science Council, Taiwan (NSC 99-2311-B182-003). The funders had no role in study design, data collection and analysis, decision to publish, or preparation of the paper.

\section{References}

[1] G. Kroemer, L. Galluzzi, and C. Brenner, "Mitochondrial membrane permeabilization in cell death," Physiological Reviews, vol. 87, no. 1, pp. 99-163, 2007.

[2] J. Estaquier, F. Vallette, J.-L. Vayssiere, and B. Mignotte, "The mitochondrial pathways of apoptosis," Advances in Experimental Medicine and Biology, vol. 942, pp. 157-183, 2012.

[3] B. Leibowitz and J. Yu, "Mitochondrial signaling in cell death via the Bcl-2 family," Cancer Biology \& Therapy, vol. 9, no. 6, pp. 417-422, 2010. 
[4] G. J. Griffiths, B. M. Corfe, P. Savory et al., "Cellular damage signals promote sequential changes at the $\mathrm{N}$-terminus and $\mathrm{BH}$ 1 domain of the pro-apoptotic protein Bak," Oncogene, vol. 20, no. 52, pp. 7668-7676, 2001.

[5] D. Westphal, G. Dewson, P. E. Czabotar, and R. M. Kluck, "Molecular biology of Bax and Bak activation and action," Biochimica et Biophysica Acta: Molecular Cell Research, vol. 1813, no. 4, pp. 521-531, 2011.

[6] X. Wang, "The expanding role of mitochondria in apoptosis," Genes and Development, vol. 15, no. 22, pp. 2922-2933, 2001.

[7] S. W. G. Tait and D. R. Green, "Mitochondria and cell death: outer membrane permeabilization and beyond," Nature Reviews Molecular Cell Biology, vol. 11, no. 9, pp. 621-632, 2010.

[8] J. M. Adams and S. Cory, "The Bcl-2 apoptotic switch in cancer development and therapy," Oncogene, vol. 26, no. 9, pp. 13241337, 2007.

[9] M. Brown and L. D. Attardi, "The role of apoptosis in cancer development and treatment response," Nature Reviews Cancer, vol. 5, no. 3, pp. 231-237, 2005.

[10] S. Fulda, "Evasion of apoptosis as a cellular stress response in cancer," International Journal of Cell Biology, vol. 2010, Article ID 370835, 6 pages, 2010.

[11] P. R. Huang, Y. M. Yeh, and T. C. V. Wang, "Potent inhibition of human telomerase by helenalin," Cancer Letters, vol. 227, no. 2, pp. 169-174, 2005.

[12] Y. J. Chen, W. Y. Sheng, P. R. Huang, and T. C. V. Wang, "Potent inhibition of human telomerase by U-73122," Journal of Biomedical Science, vol. 13, no. 5, pp. 667-674, 2006.

[13] P.-R. Huang, Y.-M. Yeh, C.-C. Pao, C.-Y. Chen, and T.-C. V. Wang, " $N$-(1-Pyrenyl) maleimide inhibits telomerase activity in a cell free system and induces apoptosis in Jurkat cells," Molecular Biology Reports, vol. 39, no. 9, pp. 8899-8905, 2012.

[14] M. E. Shawgo, S. N. Shelton, and J. D. Robertson, "Caspasemediated bak activation and cytochrome c release during intrinsic apoptotic cell death in jurkat cells," Journal of Biological Chemistry, vol. 283, no. 51, pp. 35532-35538, 2008.

[15] C. C. Yu, S. C. Lo, and T. C. V. Wang, "Telomerase is regulated by protein kinase C-zeta in human nasopharyngeal cancer cells," Biochemical Journal, vol. 355, no. 2, pp. 459-464, 2001.

[16] P. E. Czabotar, D. Westphal, G. Dewson et al., "Bax crystal structures reveal how $\mathrm{BH} 3$ domains activate Bax and nucleate its oligomerization to induce apoptosis," Cell, vol. 152, no. 3, pp. 519-531, 2013.

[17] T. Moldoyeanu, C. R. Grace, F. Llambi et al., "BID-induced structural changes in BAK promote apoptosis," Nature Structural and Molecular Biology, vol. 20, no. 5, pp. 589-597, 2013.

[18] M. Brimmell, R. Mendiola, J. Mangion, and G. Packham, "BAX frameshift mutations in cell lines derived from human haemopoietic malignancies are associated with resistance to apoptosis and microsatellite instability," Oncogene, vol. 16, no. 14, pp. 1803-1812, 1998.

[19] R. A. Winters, J. Zukowski, N. Ercal, R. H. Matthews, and D. R. Spitz, "Analysis of glutathione, glutathione disulfide, cysteine, homocysteine, and other biological thiols by highperformance liquid chromatography following derivatization by n-(1-Pyrenyl)maleimide," Analytical Biochemistry, vol. 227, no. 1, pp. 14-21, 1995.

[20] W. Wu, G. Goldstein, C. Adams, R. H. Matthews, and N. Ercal, "Separation and quantification of $\mathrm{N}$-acetyl-L-cysteine and $\mathrm{N}$ acetyl-cysteine-amide by HPLC with fluorescence detection," Biomedical Chromatography, vol. 20, no. 5, pp. 415-422, 2006.
[21] S. H. Kaufmann and W. C. Earnshaw, "Induction of apoptosis by cancer chemotherapy," Experimental Cell Research, vol. 256, no. 1, pp. 42-49, 2000.

[22] G. Dewson, T. Kratina, H. W. Sim et al., “To trigger apoptosis, bak exposes its $\mathrm{BH} 3$ domain and homodimerizes via $\mathrm{BH} 3$ : groove interactions," Molecular Cell, vol. 30, no. 3, pp. 369-380, 2008.

[23] G. Dewson, T. Kratina, P. Czabotar, C. L. Day, J. M. Adams, and R. M. Kluck, "Bak activation for apoptosis involves oligomerization of dimers via their alpha6 helices," Molecular Cell, vol. 36, no. 4, pp. 696-703, 2009.

[24] A. G. Letai, "Diagnosing and exploiting cancer's addiction to blocks in apoptosis," Nature Reviews Cancer, vol. 8, no. 2, pp. 121-132, 2008.

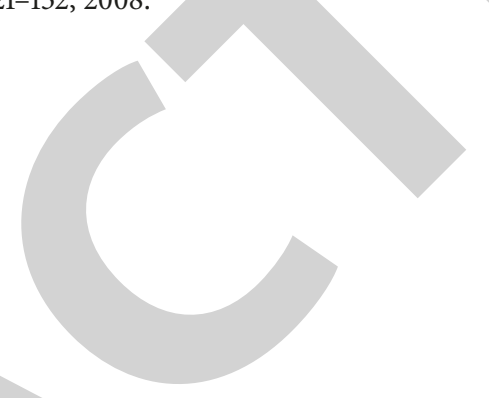

